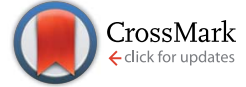

Cite this: RSC Adv., 2017, 7, 10624
Received 28th December 2016 Accepted 2nd February 2017

DOI: $10.1039 / c 6 r a 28765 d$

rsc.li/rsc-advances

\section{Fabrication of a 3D Teflon microdevice for energy free homogeneous liquid flow inside a long microchannel and its application to continuous- flow PCR}

\begin{abstract}
Kieu The Loan Trinh, $\dagger^{a}$ Wenming Wu†t $\dagger^{a}$ and Nae Yoon Lee ${ }^{\star a b}$
We introduce a simple but robust manufacturing technology for a 3D spiral Teflon microdevice, by wrapping a polytetrafluoroethylene (PTFE) tube around a poly(dimethylsiloxane) (PDMS) mold. Semiautomated sample injection inside this fluoroplastic microdevice was achieved using one disposable syringe with flow rates systemically analyzed under different inner pressures and syringe volumes. After placing the microdevice on a single hot plate at a constant temperature of $105{ }^{\circ} \mathrm{C}$, a temperature gradient was formed across the 3D spiral fluoroplastic microdevice, which is suitable for the successful amplification of two DNA targets (230 bp and $409 \mathrm{bp}$ ) from a plasmid vector. In contrast with PDMS and poly(methyl methacrylate) (PMMA) microdevices, fewer bubbles were observed in this Teflon microdevice. The effect of the Teflon surface on amplification efficiency was also analyzed. After adding different amounts of bovine serum albumin (BSA) to the same volume of polymerase chain reaction (PCR) solution, it was found that the amplification efficiency increased when BSA concentration was adjusted from 0 to $0.24 \mu \mathrm{g} \mu \mathrm{L}^{-1}$. Futhermore, we believe that this 3D spiral fluoroplastic microdevice could be a stable platform for continuous-flow PCRs.
\end{abstract}

\section{Introduction}

During the last twenty years, microfluidics has become an increasingly important technology applied in a wide range of applications. One critical issue in microfluidics is the fabrication of functionalized microdevices from new materials. Before the 2000s, most microdevices were fabricated using inorganic materials such as silicon, silicon nitride, and glass, making the microfabrication a time-consuming and labor-intensive process requiring expensive equipment. ${ }^{1}$ Although the introduction of plastics and poly(dimethylsiloxane) (PDMS) in the late 1990s has significantly increased the manufacturing efficiency of microfluidic devices, some obvious defects still exist. It is well known that most plastics are extremely vulnerable to corrosion by most organic solvents and cannot be used at temperatures higher than their glass transition temperature. ${ }^{2}$ Similarly, PDMS also has several application limitations; for example, it

\footnotetext{
aDepartment of BioNano Technology, College of BioNano Technology, Gachon University, 1342 Seongnam-daero, Sujeong-gu, Seongnam-si, Gyeonggi-do, 13120, Korea.E-mail: nylee@gachon.ac.kr

${ }^{b}$ Gachon Medical Research Institute, Gil Medical Center, Incheon 405-760, Republic of Korea

$\uparrow$ These authors contributed equally to this work.

\$ Current address: State Key Laboratory of Applied Optics, Changchun Institute of Optics, Fine Mechanics and Physics (CIOMP), Chinese Academy of Sciences, Changchun, 130033, China.
}

can easily absorb small hydrophobic permeable molecules and has a very limited solvent resistance..$^{3,4}$

To eliminate the disadvantages associated with conventional materials, some new materials have been introduced in microfabrication, of which fluoroplastics have aroused great interest owing to their superior anticorrosion property, temperature stability, low absorption of organic molecules, and good biocompatibility. ${ }^{5-8}$ Maltezos et al. built a valve structure using sacrificial printed wax molds. ${ }^{5}$ After casting and curing using liquid perfluoropolyether (SIFEL) around the wax, the wax was removed in subsequent steps. Huang et al. and Devaraju et al. fabricated a multilayer device through direct sealing of fluoroplastics, layer by layer. ${ }^{6,7}$ Rolland et al. used photo-curable perfluoropolyethers to fabricate microchips by UV exposure for $10 \mathrm{~min}^{8}$ Even if these methods show the possibility of fabricating fluoroplastic microdevices of diverse configurations, a 3D spiral microchannel is difficult to fabricate using these methods. ${ }^{5-8}$ In this study, using a method derived from a method we developed recently, we easily fabricated a 3D spiral polytetrafluoroethylene (PTFE) microdevice by wrapping a PTFE (also known as Teflon) tube around a predesigned mold, which is then applied for continuous-flow polymerase chain reaction (PCR). ${ }^{9}$

PCR microfluidics have witnessed a great development in the fabrication of microchannel. For example, computer numeric control (CNC) milling machine and photolithography are two 
prominent techniques employed by most studies depending on the types of materials used for microdevice construction..$^{10,11}$ Such methods require bulky and expensive instruments as well as complicated procedures that may not be well applied for most modestly equipped laboratories. Furthermore, major existing microscale PCR platforms still face with developing effective operating systems with minimal accessories, which is mostly hampered by using bulky syringe pumps for sample injection and multiple heaters for temperature control. ${ }^{10-12}$ With the ultimate purpose of realizing a portable device for PCR, this study introduces a simple and cost-effective approach for the fabrication of the microdevice by using Teflon together with minimizing the required accessories, demonstrating remarkable efficiency of nucleic acids amplification.

Using this system, problems such as bubble formation were successfully resolved. During high temperature processing (i.e., DNA denaturing during PCR) inside microdevices fabricated from PDMS and poly(methyl methacrylate) (PMMA), bubbles can easily form and disturb the microprocessing. Our previous research involved formulating a systematic procedure to solve this problem by using mineral oil. ${ }^{13}$ General approaches for suppressing bubbles inside a microdevice are complicated. ${ }^{13}$ Nevertheless, in the 3D spiral PTFE microdevice presented here, neither any operation nor special material is needed to suppress bubble formation under high temperatures. We hypothesize this because fewer surface pores exist in a 3D spiral PTFE microdevice in contrast to a PDMS microdevice, where it is difficult to suppress bubbles during a PCR process. ${ }^{13}$ Obviously, manufacturing steps (e.g., plasma treatment, interface chemical treatment, and device sealing) that may generate unexpected surface pores in conventional technologies such as CNC micro milling, photolithography, and laser ablation, are eliminated here. Moreover, it is evident that the glass transition temperature of PTFE is also much higher than most plastics such as PMMA. Therefore, our method provides a relatively simple solution for bubble suppression in microdevices under high temperatures.

The usability of any microfluidic device depends on the range of practical applications wherein it can be utilized. ${ }^{\mathbf{1 4 , 1 5}}$ One drawback restricting microfluidic application is its bulky apparatus. For example, most microfluidic devices today require complex and large pumping accessories to achieve sample transport through microchannels. ${ }^{16-19}$ This conflicts with the purpose of achieving a convenient and portable process expected from using a microdevice. Even if various selfactivated pumping systems have been applied to microfluidic devices, most of these pumps have strict requirements related to the surface tension of the microfluidic devices. ${ }^{20-22}$ Further, the pumping force is relatively weak to stably transport a sample over long distances under high temperatures. In our 3D spiral PTFE microdevice, we tried to incorporate a self-contained gas permeable automatic pumping arrangement instead of using a bulky pumping apparatus. ${ }^{23,24}$ The self-activated system is strong enough to handle continuous-flow PCR without being interrupted by high denaturation temperatures and drive samples to long distances. Moreover, the flow velocity is readily controlled by changing the operation parameters. The flow velocity of this self-activated pump is much more homogeneous than other self-activated pumps such as capillary pumps. ${ }^{25}$ Owing to this, the use of an external micropump is eliminated while efficiently maintaining sample transport inside the 3D spiral PTFE microdevice, using only one hot plate for the required thermal cycle between $95{ }^{\circ} \mathrm{C}$ and $58^{\circ} \mathrm{C}$.

\section{Principles}

\section{Principles of temperature control}

In this research, annealing and extension were performed at the same temperature by using a 3D structure of the PTFE microdevice. The temperature control mechanism here was similar to that of our previous research, where low heat-conductive PDMS was applied to stabilize the temperature gradient between the bottom and top surfaces of the microdevice. ${ }^{26} \mathrm{~A}$ hot plate system was used for controlling the denaturation temperature, and the height of the PDMS mold was used to control the gap between denaturation and annealing/extension temperatures. The bottom surface of the PDMS mold corresponded to the denaturation temperature, while the top surface corresponded to the annealing/extension temperatures.

A theoretical derivation for estimating the top interfacial temperature and height of the PDMS mold has been systemically studied in our previous research. ${ }^{26}$ The temperature gradient inside the PDMS mold was stabilized by two types of heat transfer theories: first, free convection heat transfer in a large room and second, heat conduction within a microdevice. Three types of thermal resistance were involved during the heat transfer. After thermal equilibrium, the temperature on the surfaces of the PDMS mold can be calculated by the following equation:

$$
\frac{t_{\mathrm{s}}^{\prime}-t_{\mathrm{rt}}}{t^{\prime \prime}{ }_{\mathrm{s}}-t_{\mathrm{s}}^{\prime}}=\frac{R_{\mathrm{t}}}{R_{\mathrm{m}}+R_{\mathrm{f}}}
$$

where $R_{\mathrm{f}}$ is the heat transfer contact resistance between the microdevice and heater; $R_{\mathrm{m}}$ is the heat conduction resistance inside the PDMS mold; $R_{\mathrm{t}}$ is the natural heat convection resistance between the topmost PDMS surface and the atmosphere; $t^{\prime}{ }_{\mathrm{s}}$ is temperature of the top surface of the PDMS mold; $t^{\prime \prime}{ }_{\mathrm{s}}$ is the temperature of the bottom surface of the PDMS mold, which is almost the same as that of the hot plate; and $t_{\mathrm{rt}}$ is the room temperature. For specialized microdevices, $R_{\mathrm{f}}, t^{\prime \prime} \mathrm{s}$, and $t_{\mathrm{rt}}$ can be considered constant, where as $R_{\mathrm{m}}$ can be calculated using the following equation:

$$
\frac{R_{\mathrm{m}}=\varphi_{\mathrm{PDMS}}}{\gamma_{\mathrm{PDMS}}}
$$

where $\varphi_{\text {PDMS }}$ is the thickness of the bottom PDMS layer, and $\gamma_{\mathrm{PDMS}}$ is the thermal conductivity of PDMS $\left(0.16 \mathrm{~W} \mathrm{~K}^{-1} \mathrm{~m}^{-1}\right) \cdot R_{\mathrm{t}}$ is virtually determined by the thickness parameter of the PDMS mold. ${ }^{26}$ Thus, the appropriate thickness of the PDMS mold can be accurately derived from the required denaturation and annealing/extension temperatures of any specialized PCRs, and in return, the thickness is also adequate for controlling the desired temperature on the top surface of the microdevice to 
achieve both denaturation and annealing/extension temperatures during thermal cycling using a single heater.

\section{Principles of flow control}

A liquid sample is delivered inside a microchannel via semiautomated fluid movement owing to air permeability from the gas-permeable fluidic conduit to the atmosphere. In our earlier studies, we found this type of self-actuated semi-automatic flow mechanism for transporting liquids inside a long microchannel (approximately $2 \mathrm{~m}$ long) fabricated using gas-permeable materials such as PDMS and silicone tubes. ${ }^{23}$ However, the flow velocity gradually decreased with time, which eventually required the use of a spiral microchannel to precisely control the reaction temperature for performing PCR. This complicated the design and fabrication of the microdevice, and control of the retention time at each temperature regime was difficult. To overcome these problems, we have recently introduced an improved pumping strategy inside a serpentine channel instead of the spiral channel by changing the material from PDMS to PMMA. Without using an external power source, we established a stable flow rate over a long distance microchannel $(1.25 \mathrm{~m}$, $2.25 \mathrm{~m}$, and $2.75 \mathrm{~m}$ ) made of PMMA. Nevertheless, the flow stability of this type of semi-automated micropump inside a 3D microdevice has never been studied. The use of 3D microdevices is highly advantageous as PCRs inside a 3D structure could be performed by employing a single heater. Thus, in this study, we further analyze the performance of the semiautomated micropump inside a 3D microdevice fabricated using PTFE tube.

Fig. 1 shows the schematic illustrations of the semiautomated sample injection mechanism proposed in this study. For actuation, a disposable syringe was connected to the inlet of the gas-impermeable PTFE microchannel, with the outlet connected by a gas-permeable silicone tube, which was blunt-ended by using a commercial clamp. As shown in Fig. 1, $P_{1}$ and $P_{2}$ represent the air pressures in the posterior and anterior ends of the sample plug, respectively, whereas $P_{g}$ represents the pressure gradient imposed on the sample plug. $V_{1}$ represents the summed volumes of air contained in the syringe and in the posterior end of the sample plug. $V_{2}$

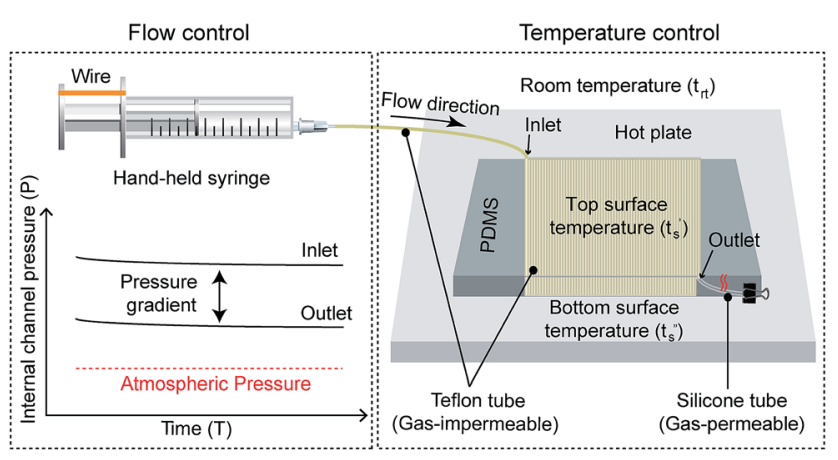

Fig. 1 Schematic illustrations demonstrating semi-automated pump to achieve homogeneous sample flow inside a PTFE microchannel employing one hot plate. represents the volume of the air contained in the anterior end of the sample plug. $S$ represents the cross-sectional area of the inner channel and can be calculated as $S=\pi r^{2}$, where $r$ is the inner diameter of the PTFE tube (ID $=0.3 \mathrm{~mm}$ ).

When the air captured inside the fluidic conduit is compressed by the syringe piston, the pressure exerted on the compressed air inside a closed fluidic conduit becomes higher than the atmospheric pressure. The pressure can be calculated by the ideal gas law, $P V=n R T$, where $n$ is the number of moles of gas, $T$ is the Kelvin temperature, and $R$ is the gas constant. Owing to much larger volume of syringe compared to that of the fluidic circuit, $V_{1}$ can be assumed to be constant during sample transport. Thus, $P_{1}$ could be considered constant because the number of moles of gas in the posterior end of the sample plug, $n_{1}$, is also constant. For the anterior end of the sample plug, it is noted that the decrease of its volume with respect to time $\left(\Delta V_{2} /\right.$ $\Delta t)$ is equal to the decrease in its number of moles with respect to time $\left(\Delta n_{2} / \Delta t\right)$ resulting from air permeation along the length of the silicone tube..$^{24} \mathrm{As}$ a result, $P_{2}$ is also constant. In addition, $P_{\mathrm{g}}$ is also constant during sample flow because $P_{\mathrm{g}}=P_{1}-P_{2}$. Based on the Hagen-Poiseuille equation, $P_{\mathrm{g}}$ is proportional to the flow rate of liquid $(u)$, the length of liquid plug $(L)$, and liquid viscosity $(\mu)$, but inversely proportional to the square of the channel diameter $(r)$. Because $L, \mu$, and $r$ are constant for any specific 3D serpentine PTFE microchannel, $u$ can be considered as constant during sample transport. Although gravity can also affect the flow velocity inside the 3D microchannel, it can be neglected because the volume of sample flowing along the gravity direction as well as against gravity direction can always be considered as constant with the lapse of time. As a result, a stable flow can be obtained inside a 3D PTFE microchannel, similar to that in the 2D PMMA microchannel in our previous research. ${ }^{25}$

\section{Experimental method}

\section{Fabrication of PTFE microdevice}

The inner diameter, outer diameter, and wall thickness of the PTFE tube were $0.3 \mathrm{~mm}, 0.7 \mathrm{~mm}$, and $0.2 \mathrm{~mm}$, respectively. The PTFE microdevice was fabricated by wrapping a $2 \mathrm{~m}$ long tube around a PDMS mold prepared at a 10:1 ratio of the prepolymer and curing agent. The width and height of the final microdevice were $3 \mathrm{~cm}$ and $1 \mathrm{~cm}$, respectively, to achieve a total of 25 thermal cycles. The wrapped tubes were glued and fixed tight. Afterward, a $13 \mathrm{~cm}$ long silicone tube (ID $=0.64 \mathrm{~mm}$, OD $=1.19 \mathrm{~mm}$ ) was connected to the outlet of the PTFE microchannel.

\section{Flow control}

A $25 \mathrm{~mL}$ syringe was used for sample introduction and transport inside the microchannel. Four flow tests were conducted using initial internal pressures of approximately $1.25 \mathrm{~atm}, 1.47 \mathrm{~atm}$, $1.67 \mathrm{~atm}$, and $2.5 \mathrm{~atm}$, by pushing the piston from the initial graduation of 25 to $20,17,15$, and 10, respectively. Every experiment was performed thrice to verify reproducibility. The process of fluid manipulation was similar to that in our 
previous report. ${ }^{23,24}$ First, the silicone tube that connects the outlet of the PTFE microdevice was blocked by a clamp. Second, initial pressures were applied from the $25 \mathrm{~mL}$ syringe that connects the inlet of the microdevice and maintained with a piece of iron wire. Finally, the flow velocity and stability were analyzed by varying the aforementioned initial pressures. More pictures of the operational steps can be found in our previous research. ${ }^{23,24}$

\section{On-chip continuous-flow PCR}

A commercially available pGEM-3Zf $(+)$ plasmid vector $(10 \mathrm{ng}$ $\mu \mathrm{L}^{-1}$ ) was diluted and added to the PCR reagent to make a final template concentration of $5 \mathrm{ng}$ per reaction, while a synthetic pGEM-T vector inserted with a 409 bp gene fragment of the TTF1 target gene (20-50 ng $\mu \mathrm{L}^{-1}$ of concentration in stock solution) was further diluted and added to the PCR reagent to make the final template concentration of 3-4.5 ng per reaction. The primer sequences for amplifying a $230 \mathrm{bp}$ gene fragment in the pGEM-3Zf $(+)$ plasmid vector were as follows: $5^{\prime}$-CCG GCG AAC GTG GCG AGA AAG GAA GGG AAG AAA GC- $3^{\prime}$ (forward) and 5'TCG CCT TGC AGC ACA TCC CCC TTT CGC CAG C-3' (reverse). The primer sequences for amplifying the specific sequence in TTF-1 were as follows: $5^{\prime}$-CAC CTT GCT ATA CGG TCG GAC-3' (forward) and 5'-AGA GTC CAG AGC CAT GTC AGC-3' (reverse). The $25 \mu \mathrm{L}$ sample plug contained a mixture of the DNA template and PCR reagents such as a green-colored buffer, $0.16 \mathrm{mM}$ dNTPs mixture, $1.5 \mu \mathrm{g}$ of BSA, $0.5 \mu \mathrm{M}$ of forward and reverse

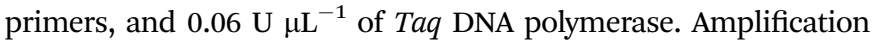
was performed for 25 cycles. Before the introduction of the sample plug, the internal microchannel was first passivated with $600 \mu \mathrm{L}$ of BSA solution $\left(1.5 \mu \mathrm{g} \mu \mathrm{L}^{-1}\right)$. DNA amplicons were detected using agarose gel electrophoresis at $254 \mathrm{~nm}$ using a UV transilluminator. For positive control, PCR was also performed using a thermocycler for both targets (230 and $409 \mathrm{bp}$ ) under the same temperature conditions. Denaturation and annealing/extension were performed at $95{ }^{\circ} \mathrm{C}$ and $58{ }^{\circ} \mathrm{C}$, respectively for 25 thermal cycles.

\section{Results and discussions}

\section{Temperature control of on-chip PCR}

In order to reduce the number of heaters for PCR assays as mentioned in the "Introduction", a single hot plate was used to control the temperature for the on-chip PCR (Fig. 2a). Also, the

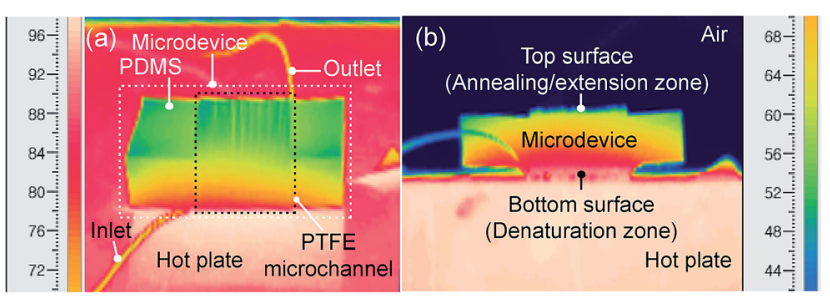

Fig. 2 Results of temperature measurement. (a) IR camera image of both the top and side view of the 3D PTFE microdevice. (b) Side view of PTFE tube wrapped around PDMS mold ( $1 \mathrm{~cm}$ high) to form temperature gradient in vertical direction. two-temperature PCR process was introduced for DNA amplifications. For temperature control, the hot plate was kept at approximately $105{ }^{\circ} \mathrm{C}$, with the 3D Teflon microdevice being placed atop. In that way, a positive temperature gradient was applied on the microdevice and generated from the top to the bottom surface (Fig. 2b). In detail, owing to the existence of contact heat resistance (as demonstrated in "Principles section"), the top and bottom surface temperature of the PTFE microdevice were $58.0 \pm 0.3{ }^{\circ} \mathrm{C}(\mathrm{CV}=0.4 \%(n=10))$ and $95.5 \pm$ $1.1{ }^{\circ} \mathrm{C}(\mathrm{CV}=1.2 \%(n=10))$ respectively, resulted from the low thermal conductivity of PTFE $\left(0.25 \mathrm{~W} \mathrm{~m}^{-1} \mathrm{~K}^{-1}\right)$ as well as PDMS mold $\left(0.17 \mathrm{~W} \mathrm{~m}^{-1} \mathrm{~K}^{-1}\right)$. Moreover, different annealing/ extension temperatures could be obtained freely by varying the thickness of the PDMS mold. In addition, stable temperature was maintained for up to $1 \mathrm{~h}$, which was sufficient for running the on-chip PCR.

\section{Flow control of on-chip PCR}

Flow analyses were performed in two ways. First, the volume of syringe was varied from (i) $25 \mathrm{~mL}$ to $15 \mathrm{~mL}$ and (ii) $60 \mathrm{~mL}$ to 50 mL. As shown in Fig. 3, the flow rates were relatively different despite the same difference of $10 \mathrm{~mL}$ applied for the two cases. For the $60 \mathrm{~mL}$ syringe volume in Fig. 3, the flow rate of the earlier several cycles was obviously faster than the latter cycles, with retention time increasing from 143 to $159 \mathrm{~s}$. By changing the initial syringe volume to $25 \mathrm{~mL}$, it seems that the smaller syringe volume $(25 \mathrm{~mL})$ displayed an improved flowing stability as compared with the bigger one $(60 \mathrm{~mL})$, with the average time of each cycle reduced to $50 \mathrm{~s}$. From this experiment, it is concluded that the initial volume of syringe can influence both
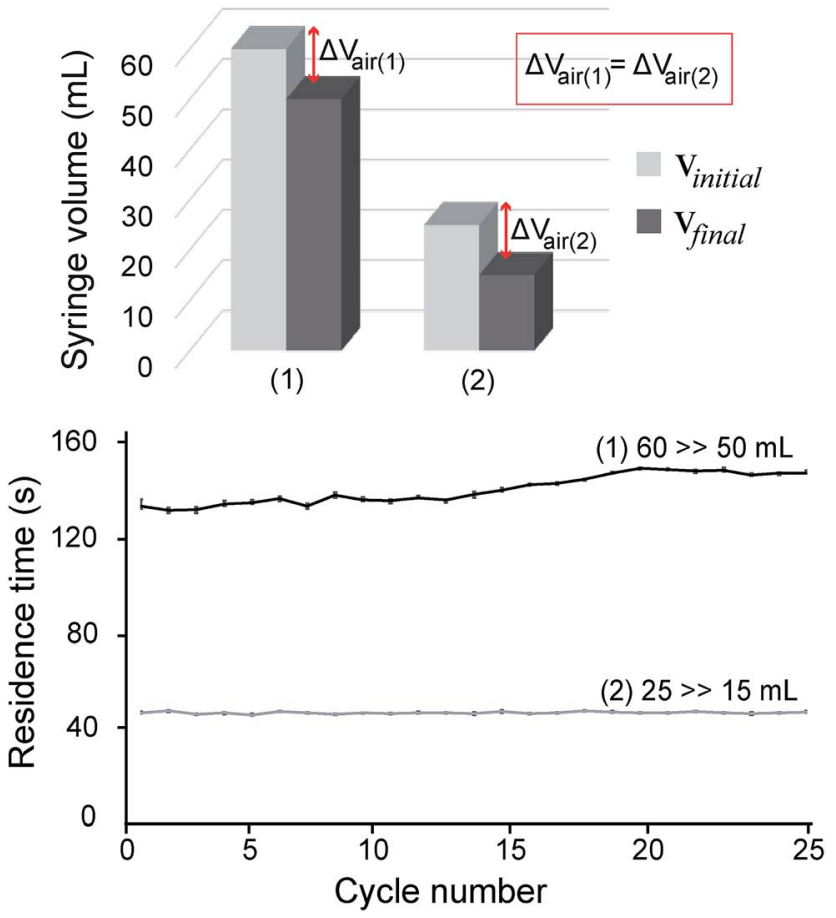

Fig. 3 Comparison of flow rates using different volumes of syringes (60 mL vs. $25 \mathrm{~mL}$ ). 
flow rate and flow stability of the liquid inside the PTFE microchannel. From our analysis, the reason is because a higher internal pressure can produce more stable air diffusion/permeation through the outlet silicone tube, which further caused a more steady flow rate inside the PTFE tube. Based on these results, we chose the $25 \mathrm{~mL}$ syringe for the flow control during the on-chip PCR. The standard deviations were calculated based on three repeated experiments with a residence time of 25 cycles. The pumping velocities of different syringe volumes were statistically different from each other $(P<$ 0.005).

Second, the effect of initial pressure on the flow rate was analyzed using the $25 \mathrm{~mL}$ syringe. The initial pressures were calculated to be approximately $2.5 \mathrm{~atm}, 1.6 \mathrm{~atm}, 1.47 \mathrm{~atm}$, and 1.25 atm, respectively when air inside the syringe was compressed to a graduation of $10 \mathrm{~mL}, 15 \mathrm{~mL}, 17 \mathrm{~mL}$, and $20 \mathrm{~mL}$, respectively. As shown in Fig. 4, when the initial pressure was lowest (1.25 atm), it displayed the slowest retention time of approximately $140 \mathrm{~s}$ for each cycle, which required nearly $70 \mathrm{~min}$ for sample flowing from the inlet to the outlet of the microchannel. In contrast, the highest initial pressure (2.5 atm) displayed the fastest flow with an average retention time of $29 \mathrm{~s}$, which required approximately $15 \mathrm{~min}$ for sample flowing from the inlet to the outlet of the microdevice. The retention time for each cycle took approximately $95 \mathrm{~s}$ and $50 \mathrm{~s}$ for the initial pressures of 1.47 and 1.6 atm, respectively, requiring approximately $47 \mathrm{~min}$ and $25 \mathrm{~min}$, respectively. Obviously, a higher initial pressure can strongly push the liquid sample through the outlet as compared with a lower one, which further resulted in
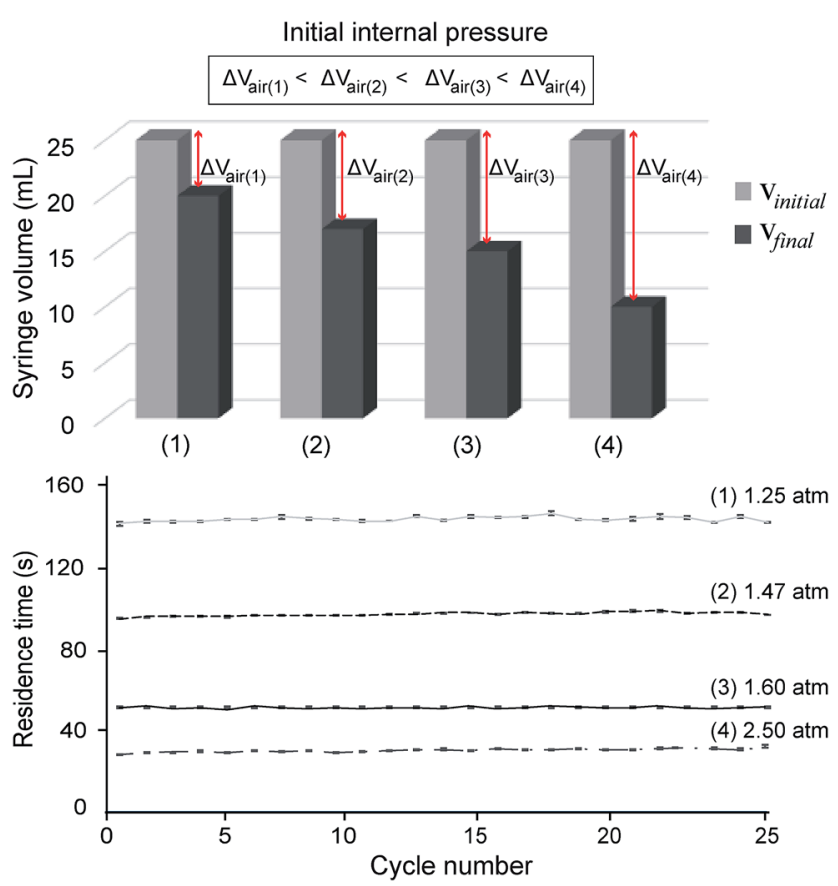

Fig. 4 Results showing flow rate changes with respect to initial pressure when $25 \mathrm{~mL}$ syringe was used. The line graph shows the flow rates inside the microchannel regarding the different initial pressure values (line 1 - 1.25 atm, line $2-1.47$ atm, line $3-1.6$ atm, line $4-2.5$ atm). a faster flow rate inside the PTFE tube. Also, all experiments were conducted to control the liquid flow rate inside the long and rounded microchannel without using a bulky syringe pump. Based on these results, it can be concluded that flow rates could be readily controlled by adjusting the initial pressure applied on the PCR system. The retention time decreased with increase in the initial pressure. The experiments were repeated three times for reproducibility. The pumping velocities at different endpoint volumes were statistically different from each other $(P<0.005)$.

\section{Continuous-flow PCR (1): effect of BSA passivation inside the 3D PTFE microreactor}

Lane 1 shows the result of positive control obtained on a thermocycler. Lanes 2-7 show amplification results obtained on microdevice when the concentrations of BSA in the PCR mixture are $0,0.06,0.12,0.18,0.24$, and $0.3 \mu \mathrm{g} \mu \mathrm{L}^{-1}$, respectively. Lane $M$ is a 100 bp DNA size marker. The relative intensity scales of the target amplicons are shown below the gel image.

For microfluidic PCRs, the internal surface of microchannel is usually modified to decrease the nonspecific adsorption of the mixture component onto the microchannel. After adding BSA into the PCR mixture, dynamic passivation of the microchannel was achieved. The function of the BSA was to decrease the adsorption of Taq DNA polymerase contained in the PCR mixture. While the sample flows, BSA is adsorbed onto the inner surface of the PTFE microchannel. As BSA readily adsorbs onto the microchannel, it prevents nonspecific adsorption of PCR reagents such as Taq DNA polymerase, DNA template, dNTPs, and primers onto the inner wall of the PTFE microchannel.

In this study, the effect of BSA dynamic passivation of inner surface of the PTFE microchannel was examined during continuous-flow PCR. Fig. 5 shows the effect of BSA concentrations on the results of PCR. Although the surface of PTFE microchannel was smooth, the DNA amplification was not successful when no BSA was added to the PCR reagent (lane 2). However, the PCR performance was significantly enhanced with

(a)

(b)
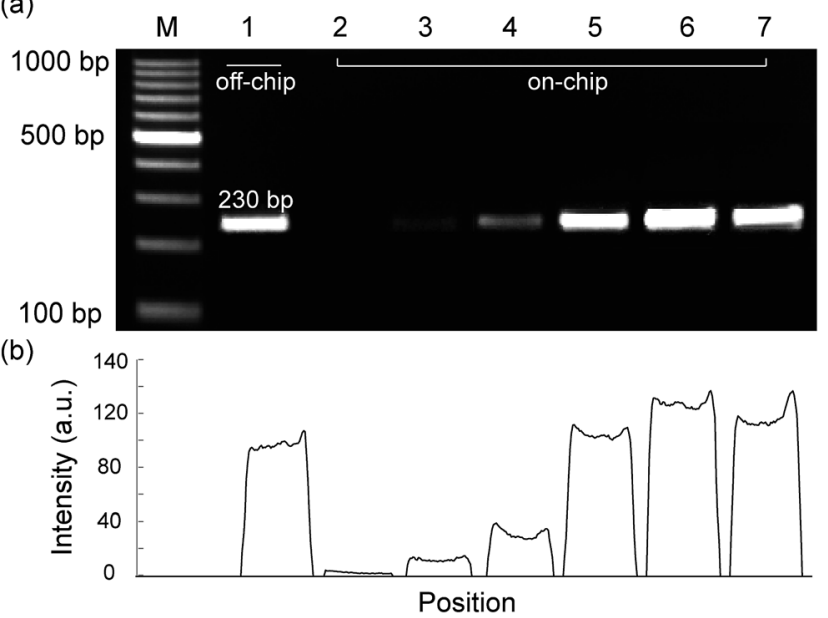

Fig. 5 Effect of BSA on continuous-flow PCRs using PTFE microdevice. 
the addition of BSA (lanes 3 to 7). As a result, BSA is needed to improve the efficiency of on-chip PCR as it helps to prevent nonspecific adsorption of PCR reagents onto the microchannel. ${ }^{27}$ Moreover, when the concentration of BSA increased, the efficiency of PCR was also enhanced. The concentrations of BSA inside the PCR mixtures were varied from $0.06 \mu \mathrm{g} \mu \mathrm{L}^{-1}$ (lane 1 (off-chip)) and $0-0.3 \mu \mathrm{g} \mu \mathrm{L}^{-1}$ (lanes $2-7$ (on-chip)). The amplification intensities of the target gene were analyzed to be 71.9\% (lane 1), 1.6\% (lane 2), 9.0\% (lane 3), 23.1\% (lane 4), $79.3 \%$ (lane 5), 100\% (lane 6), and 90.5\% (lane 7), respectively, when the intensity of $0.24 \mu \mathrm{g} \mu \mathrm{L}^{-1}$ (lane 6) was set at $100 \%$. Because the tube utilized for conventional thermocycler was not passivated by BSA, it is significant that the passivated device here can produce a higher amplification efficiency than the conventional cycler. Although the BSA concentration in lane 7 was higher than that in lane 6 , the efficiency of amplification was not improved further, probably because the BSA concentration already reached the saturation of dynamic passivation. Based on these results, we can conclude that a BSA lowers than $0.24 \mu \mathrm{g} \mu \mathrm{L}^{-1}$ can improve the result of the continuous-flow PCR inside the PTFE microchannel.

\section{Flow-through PCR (2): amplification of targets with clinical significance}

After analysis of the sample flow and optimization of the BSA concentration inside the PTFE microchannel, the microdevice was applied for the amplification of two target DNAs. The pressure was controlled at 1.66 atm by pressing the syringe piston from $25 \mathrm{~mL}$ to $15 \mathrm{~mL}$, and the concentration of BSA was controlled at $0.24 \mu \mathrm{g} \mu \mathrm{L}^{-1}$. One hot plate was used as a source of heat, and denaturation and annealing/extension were performed at $95{ }^{\circ} \mathrm{C}$ and $58{ }^{\circ} \mathrm{C}$, respectively. Because the PTFE microdevice has a smooth channel surface, bubble formation inside the microdevice was negligible despite the high temperature applied. As shown in Fig. 6, two targets of DNA
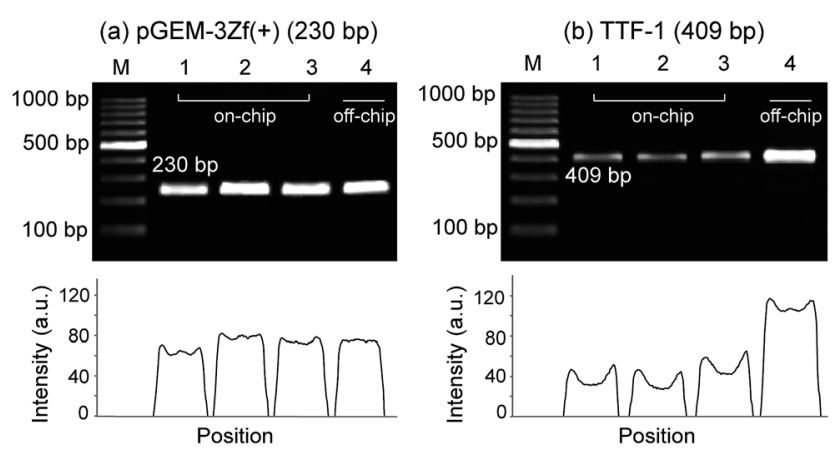

Fig. 6 Results of DNA amplification using PTFE microdevice. (a) Results of agarose gel electrophoresis for 230 bp gene fragments. Lanes 1, 2, and 3 show the amplified targets using a PTFE microdevice and lane 4 shows the amplified target obtained using a thermocycler. (b) Results of agarose gel electrophoresis for TTF-1 target gene (409 bp) amplified on PTFE microdevice (lanes 1-3) and when using a thermocycler (lane 4). Lane $M$ is a 100 bp DNA size marker. The relative intensity scales of the target amplicons are shown below each gel image.
(TTF-1 target gene of 409 bp and pGEM-3Zf(+) of $230 \mathrm{bp}$ ) were successfully amplified within 25 min after 25 thermal cycles. The obtained target amplicons were exceptionally strong as to be comparable to those obtained using a thermocycler. For example, in Fig. 6a, the amplicon of the $230 \mathrm{bp}$ target gene displayed a higher intensity as compared with that obtained using a thermocycler. The average intensities of the amplicons were $80.2 \%, 100 \%, 92.8 \%$, and $88.7 \%$, respectively, when the intensity of the on-chip PCR (lane 2) was set at $100 \%$. The adsorption of the target and Taq DNA polymerase on polypropylene tube may decrease its PCR efficiency to be lower than a surface passivated device. Although the result of $409 \mathrm{bp}$ target gene amplified using the microdevice was not as effective as those of the $230 \mathrm{bp}$ target (the intensities of the amplified targets obtained using the microdevice shown in Fig. 6a were $34.2 \%, 31.2 \%$, and $44.7 \%$, respectively, of that obtained using a thermocycler), probably because of the relatively longer gene fragment, the target amplicons were clearly distinguishable. This confirms the reliability and reproducibility of the PTFE microdevice used in this study.

\section{Conclusion}

In this study, we introduced a simple continuous-flow PCR system constructed using the PTFE tube itself. The most important point of this study was that the new PCR system was able to control the flow rate stably without the need for an external mechanical pump. By reducing the number of heaters and removing external pumps, energy can be saved and system portability, which is currently a hot issue for system miniaturization, is enhanced. PTFE was demonstrated as a choice of material for fabricating the PCR microdevice owing to its low surface energy, reduced bubble formation, and most of all, simple fabrication procedure.

\section{Acknowledgements}

This research was support by the Basic Science Research Program through the National Research Foundation of Korea (NRF) funded by the Ministry of Science, ICT \& Future Planning (2014R1A1A3051319) and GRRC program of Gyeonggi province (GRRC Gachon 2016-B01).

\section{References}

1 A. Taberham, M. Kraft, M. Mowlem and H. Morgan, J. Micromech. Microeng., 2008, 18, 64011.

2 Y. Sun, M. V. D. Satyanarayan, N. T. Nguyen and Y. C. Kwok, Sens. Actuators, B, 2008, 130, 836.

3 W. Wu, J. Wu, J. H. Kim and N. Y. Lee, Lab Chip, 2015, 15, 2819.

4 R. Dangla, F. Gallaire and C. N. Baroud, Lab Chip, 2010, 10, 2972.

5 G. Maltezos, E. Garcia, G. Hanrahan, F. A. Gomez, S. Vyawhare, R. M. Van Dam, Y. Chena and A. Scherer, Lab Chip, 2007, 7, 1209. 
6 N. S. G. K. Devaraju and M. A. Unger, Lab Chip, 2011, 11, 1962.

7 Y. Huang, P. Castrataro, C. Lee and S. R. Quake, Lab Chip, 2007, 7, 24.

8 J. P. Rolland, R. M. Van Dam, D. A. Schorzman, S. R. Quake and J. M. DeSimone, J. Am. Chem. Soc., 2004, 126, 2322.

9 W. Wu, K. T. L. Trinh and N. Y. Lee, Analyst, 2015, 140, 1416. 10 W. Wu, K. T. L. Trinh and N. Y. Lee, RSC Adv., 2015, 5, 12071. 11 J. M. Bienvenue, L. A. Legendre, J. P. Ferrance and J. P. Landers, Forensic Sci. Int.: Genet., 2010, 4, 178.

12 B. Shu, C. Zhang and D. Xing, Anal. Chim. Acta, 2014, 826, 51. 13 W. Wu, K. T. Kang and N. Y. Lee, Analyst, 2011, 136, 2287.

14 W. Zheng, Z. Wang, W. Zhang and X. Jiang, Lab Chip, 2010, 10, 2906.

15 G. M. Whitesides, Nature, 2006, 442, 368.

16 G. M. Whitesides, Lab Chip, 2013, 13, 11.
17 I. D. Johnston, M. C. Tracey, J. B. Davis and C. K. L. Tan, Lab Chip, 2005, 5, 318.

18 C. Lui, S. Stelick, N. Cady and C. Batt, Lab Chip, 2010, 10, 74. 19 H. Lai and A. Folch, Lab Chip, 2011, 11, 336.

20 J. Kim, J. Baek, K. Lee, Y. Park, K. Sun, T. Lee and S. Lee, Lab Chip, 2006, 6, 1091.

21 E. Berthier and D. J. Beebe, Lab Chip, 2007, 7, 1475.

22 D. Juncker, H. Schmid, U. Drechsler, H. Wolf, M. Wolf, B. Michel, N. de Rooij and E. Delamarche, Anal. Chem., 2002, 74, 6139.

23 W. Wu, K. T. L. Trinh and N. Y. Lee, Analyst, 2012, 137, 983. $24 \mathrm{~W}$. Wu and N. Y. Lee, Sens. Actuators, B, 2013, 181, 756.

25 W. Wu and A. Manz, RSC Adv., 2015, 5, 70737.

26 W. Wu and N. Y. Lee, Anal. Bioanal. Chem., 2011, 400, 2053. 27 R. Kodzius, K. Xiao, J. Wu, X. Yi, X. Gong, I. G. Foulds and W. Wen, Sens. Actuators, B, 2012, 161, 349. 